\title{
Tissue Iron Promotes Wound Repair via M2 Macrophage Polarization and the Chemokine (C-C Motif) Ligands 17 and 22
}

Holly N. Wilkinson, * Elizabeth R. Roberts, * Amber R. Stafford, * Kayleigh L. Banyard, * Paolo Matteucci, ${ }^{\dagger}$ Kimberly A. Mace, and Matthew J. Hardman*

From the Centre for Atherothrombosis and Metabolic Disease,* Hull York Medical School, University of Hull, Hull; the Hull and East Yorkshire Hospitals National Health Service Trust, ${ }^{\dagger}$ Hull; and the Faculty of Biology, Medicine and Health, ${ }^{\ddagger}$ The University of Manchester, Manchester, United Kingdom

Accepted for publication

July $25,2019$.

Address correspondence to Matthew J. Hardman, Ph.D., Castle Hill Hospital, University of Hull, Cottingham Rd., Daisy Bldg, Second Floor, Hull HU16 5JQ, United Kingdom. E-mail: m.hardman@hull.ac.uk.

\begin{abstract}
Macrophages are important for effective iron recycling and erythropoiesis, but they also play a crucial role in wound healing, orchestrating tissue repair. Recently, we demonstrated a significant accumulation of iron in healing wounds and a requirement of iron for effective repair. Herein, we sought to determine the influence of iron on macrophage function in the context of wound healing. Interestingly, wound macrophages extensively sequestered iron throughout healing, associated with a prohealing M2 phenotype. In delayed healing diabetic mouse wounds, both macrophage polarization and iron sequestration were impaired. In vitro studies revealed that iron promotes differentiation, while skewing macrophages toward a hypersecretory M2-like polarization state. These macrophages produced high levels of chemokine ( $\mathrm{C}-\mathrm{C}$ motif) ligands 17 and 22, promoting wound reepithelialization and extracellular matrix deposition in a human ex vivo wound healing model. Together, these findings reveal a novel, unappreciated role for iron in modulating macrophage behavior to promote subsequent wound repair. These findings support therapeutic evaluation of iron use to promote wound healing in the clinic. (Am J Pathol 2019, 189: 2196-2208; https://doi.org/ 10.1016/j.ajpath.2019.07.015)
\end{abstract}

Macrophages play a crucial role in orchestrating the wound healing response, fulfilling prodigiously versatile functions. ${ }^{1}$ Evolutionarily conserved receptors allow macrophages to detect and phagocytose pathogenic organisms in early repair, ${ }^{2,3}$ whereas their high plasticity makes them master effector cells, able to regulate inflammation and drive tissue repair. ${ }^{4}$ Most, but not all, wound macrophages originate from circulating bone marrow-derived monocytes. Once at the site of injury, these cells undergo marked, yet transient, morphologic and behavioral changes in response to the local wound milieu. ${ }^{5,6}$ Traditionally, wound-derived macrophages are categorized into two main subsets: classically activated (M1 stimulated) and alternatively activated (M2 stimulated $^{1}$ ). However, the M2 repertoire has now expanded, ${ }^{7}$ with macrophage polarization currently understood to be a spectrum of phenotypes largely governed by tissue status and environmental stimuli. ${ }^{8}$
M1-stimulated macrophages are induced in early wound repair by proinflammatory mediators, such as lipopolysaccharides and interferon- $\gamma$, driving inflammation by secreting reactive oxygen species and proinflammatory cytokines (eg, IL-1, IL-6, and tumor necrosis factor- $\alpha^{9}$ ). Wound progression is then characterized by a switch to alternative activation (M2), partly mediated by efferocytosis. ${ }^{10}$ M2-stimulated macrophages express anti-inflammatory markers (eg, IL-4, IL-10, IL-13, and transforming growth factor- $\beta 1^{11}$ ) and produce arginase, a key mediator of effective wound repair. ${ }^{12}$ Furthermore, M2-polarized macrophages produce a myriad of growth factors to facilitate reepithelialization, fibroplasia, and angiogenesis. ${ }^{13}$ Together, these mechanisms promote the

Supported by an MRC (UK) industrial CASE Ph.D. Studentship grant MR/M016307/1 (M.J.H.).

Disclosures: None declared. 
scavenging of debris, bacteria, and proinflammatory cells; and they orchestrate reparative processes to allow wound resolution. ${ }^{1}$

There is a clear existing association between iron and macrophages in the context of erythropoiesis. Herein, Kupffer cells and nurse macrophages coordinate iron to support erythropoiesis, ${ }^{14}$ and splenic macrophages crucially recycle iron from senescent erythrocytes. ${ }^{15}$ As iron is associated with heme-containing proteins for oxygen transport and metabolism, iron recycling and regulation by macrophages prevent a range of iron-mediated pathologies. ${ }^{16}$ Indeed, mice with macrophages lacking ferroportin (the only know intracellular iron exporter) become anemic, with iron loading in the spleen and liver and defective macrophage function. ${ }^{17}$ Thus, iron must be tightly regulated within the body to maintain normal physiology and avoid tissue pathology.

In the context of tissue repair, the cellular importance of iron is less clear. We have recently shown a substantial, previously unreported, accumulation of iron in the latter stages of wound healing that is attenuated in pathologic diabetic wounds. ${ }^{18}$ This supports preceding observations that abnormal iron levels are associated with impaired healing. ${ }^{19,20}$ Specifically, iron modulates fibroblast behavior to promote healing via increased extracellular matrix (ECM) deposition. ${ }^{18}$ The hypothesis of the current study is that iron plays an important role in modulating macrophage behavior to promote healing. Using a combination of in vitro and ex vivo studies, we explore the effect of iron on macrophage polarization, secretory profile, and ability to promote healing in a human ex vivo wound model. The data demonstrate an important new role for iron in mediating macrophage function and subsequent contribution to wound healing.

\section{Materials and Methods}

\section{Animal Housing and Experimentation}

Young (8- to 10-week-old) female wild-type (C57/B16), nondiabetic $\left(\mathrm{NDb} ; \mathrm{Lepr}^{-/+}\right)$, and diabetic $\left(\mathrm{Db} ; \mathrm{Lepr}^{--}\right)$mice were purchased from Envigo Ltd. (Huntingdon, Cambridgeshire, UK) and housed at the University of Manchester Biological Services Facility. Mice received food and water ad libitum and were kept under a 12-hour light/dark cycle with constant temperature and humidity. Regulated procedures were performed according to UK Home Office regulations under project license 70/8136. Two equidistant 6-mm dorsal excisional wounds were made, as described previously. ${ }^{21}$ Normal skin (day 0) and excisional wounds were collected and fixed in neutral-buffered formalin for histology.

\section{Immunohistochemistry Staining}

Paraffin sections were cut ( $5 \mu \mathrm{m}$ thick), dewaxed in xylene, and brought to water down an ethanol gradient. Antigen retrieval was performed with citrate buffer $(\mathrm{pH} \mathrm{6})$ before primary antibody incubation at $4^{\circ} \mathrm{C}$ overnight. For immunofluorescence, anti-rabbit ferritin (clone EPR3004Y), anti-mouse $\alpha$-smooth muscle actin (clone 1A4; Abcam, Cambridge, UK), and anti-rat Mac-3 (clone M3/84; BD Biosciences, Reading, UK) were used at $5 \mu \mathrm{g} / \mathrm{mL}$. Anti-rabbit keratin 14 (clone Poly19053; Biolegend, San Diego, CA), antimouse COL1A1 (clone 3G3), and anti-mouse COL3A1 (clone C-6; Santa Cruz Biotechnology, Heidelberg, Germany) were used at $1 \mu \mathrm{g} / \mathrm{mL}$. Alexa Fluor 488-conjugated (Mac-3, $\alpha-$ smooth muscle actin, and COL1A1) and Alexa Fluor 594-conjugated (ferritin, keratin 14, COL1A1, and COL3A1) secondary antibodies were used at $10 \mu \mathrm{g} / \mathrm{mL}$ for 1 hour at room temperature (all from Thermo Fisher Scientific, Paisley, UK). Slides were mounted with Mowiol 488 (Sigma-Aldrich, Dorset, UK) containing DAPI (Thermo Fisher Scientific). Images were taken on an LSM710 laser scanning confocal microscope (Carl Zeiss Ltd, Cambridge, UK) using a $20 \times$ objective and the 405-nm diode, 488-nm argon, and 561-nm Dulbecco's phosphate buffered saline lasers. Mac-3-, ferritin-, and $\alpha$-smooth muscle actin-positive cells were counted in ImageJ software version 1.8.0 (NIH, Bethesda, MD; http:// imagej.nih.gov/ij). For immunoperoxidase staining, anti-rat Mac-3 (above), anti-rabbit nitric oxide synthase 2, and antigoat arginase-1 antibodies (both from Santa Cruz Biotechnology) were used at $1 \mu \mathrm{g} / \mathrm{mL}$ and detected using appropriate VECTASTAIN ELITE ABC kits and NovaRED peroxidase substrate (Vector Laboratories, Peterborough, UK). Brightfield images were obtained on a Nikon E400 microscope (Nikon Instruments Inc., Melville, NY) and SPOT camera (SPOT Imaging, Sterling Heights, MI). Cell counts were performed in ImagePro-Plus version 6.3.0 (Media Cybernetics, Cambridge, UK).

\section{THP-1 Cell Culture}

THP-1 cells (ATCC TIB-202; ATCC, Middlesex, UK) were used as a human monocyte model. ${ }^{22}$ THP-1 cells were grown in RPMI 1640 medium (Thermo Fisher Scientific) with 10\% heat-inactivated fetal bovine serum (Thermo Fisher Scientific) and 1\% penicillin-streptomycin solution (Thermo Fisher Scientific).

\section{THP-1 Cell Differentiation Experiments}

Phorbol 12-myristate 13-acetate (PMA) was used at $200 \mathrm{ng} / \mathrm{mL}$ to induce differentiation in THP-1 cells. THP-1 cells were treated with or without PMA and iron in the form of ferric ammonium citrate (FAC) at 10 and $100 \mu \mathrm{mol} / \mathrm{L}$ concentrations. After 3 days of incubation, THP-1 cells were collected for RNA (below) to assess the expression of the differentiation markers, CD11b,CD14,CD36, and CD54, via quantitative real-time PCR. ${ }^{23,24}$

\section{THP-1 Cell Polarization Experiments}

After PMA stimulation for 3 days to induce differentiation, THP-1-derived macrophages (TDMs) were stimulated to 
M1 (interferon- $\gamma$ and lipopolysaccharide) and M2 (IL-4 and IL-13) states using reagents at $20 \mathrm{ng} / \mathrm{mL}$ concentration (all from Thermo Fisher Scientific) or left unstimulated (M0 treatment group). For these polarization experiments, TDMs were treated with FAC at the same time as adding cytokines. TDMs were collected after 24 hours for quantitative realtime PCR, immunocytochemistry (using ferritin antibody, as above), and Western blot analysis.

\section{Quantitative Real-Time PCR}

Macrophages were collected in Ambion TRIzol reagent (Thermo Fisher Scientific), and RNA was isolated using phenol/chloroform. ${ }^{25}$ RNA was purified using the Ambion PureLink Mini Kit (Thermo Fisher Scientific), as per manufacturer's instructions, and reverse transcribed with Bioscript (Bioline, London, UK) and Random Primers (Promega, Southampton, UK). Quantitative real-time PCR was performed with $2 \times$ Takyon SYBR Green mastermix (Eurogentec, Hampshire, UK) and a CFX Connect thermocycler (Bio-Rad Laboratories, Hertfordshire, UK). Primer sequences are provided in Table 1.

\section{Adhesion and Zymography}

THP-1 cell adhesion was assessed after PMA and FAC stimulation (as per differentiation experiments). Herein, wells were rinsed with Dulbecco's phosphate-buffered saline (Thermo Fisher Scientific) to remove nonadherent cells; and CellTiter 96 AQueous One Solution (Promega) was added to the wells, as per manufacturer's guidelines. Zymography was also performed on conditioned medium (CM) collected from these THP-1 cells, as described previously. ${ }^{26}$

\section{Fibroblast Matrix Preparation}

Human skin was collected from theaters at Castle Hill Hospital (Cottingham, Hull, UK) under local research ethics committee (17/SC/0220) approval and full, informed patient consent. Human dermal fibroblasts (HDFs) were isolated, as previously described. ${ }^{21} \mathrm{HDFs}$ were cultured in minimum essential medium (Gibco, Thermo Fisher Scientific) in 2\% fetal bovine serum with FAC for 11 days. Cells were removed (denuded) from the HDF-derived ECM with $20 \mathrm{mmol} / \mathrm{L}$ ammonium hydroxide (Sigma-Aldrich) in $0.5 \%$ Triton X-100 in Dulbecco's phosphate-buffered saline. Denuded ECM was treated with $10 \mu \mathrm{g} / \mathrm{mL}$ DNAse (Sigma-Aldrich) and extensively washed. THP-1 cells were seeded on this HDF-derived ECM with or without $200 \mathrm{ng} / \mathrm{mL}$ PMA, left for 3 days, and collected for differentiation assessment via quantitative realtime PCR. Immunocytochemistry and Perls Prussian Blue staining were performed on the HDF-derived ECM.

\section{Ferrozine Assay}

A ferrozine assay allowed for the detection of ferrous and ferric iron. ${ }^{27}$ Denuded HDF-derived ECM was scraped on ice in $50 \mathrm{mmol} / \mathrm{L} \mathrm{NaOH}$ containing $1 \%$ HALT protease inhibitor cocktail (Thermo Fisher Scientific). $\mathrm{HCl}(10 \mathrm{mmol} / \mathrm{L})$ was added to each lysate with $200 \mu \mathrm{L}$ iron-releasing reagent $\left(8.6 \% \mathrm{v} / \mathrm{v} \mathrm{HCl}\right.$ and $280 \mu \mathrm{mol} / \mathrm{L} \mathrm{KMnO}_{4}$ in distilled water) for 2 hours at $60^{\circ} \mathrm{C}$. Standards of known concentrations of FAC $(100 \mathrm{mmol} / \mathrm{L}, 10 \mathrm{mmol} / \mathrm{L}, 1 \mathrm{mmol} / \mathrm{L}, 100 \mu \mathrm{mol} / \mathrm{L}, 10 \mu \mathrm{mol} / \mathrm{L}$, $1 \mu \mathrm{mol} / \mathrm{L}$, and $0 \mu \mathrm{mol} / \mathrm{L}$ ) were prepared and incubated, as described. Samples were cooled at room temperature, and 50 $\mu \mathrm{L}$ iron detection reagent $(650 \mu \mathrm{mol} / \mathrm{L}$ ferrozine, $650 \mu \mathrm{mol} / \mathrm{L}$ neocuproine, $2.8 \mathrm{mmol} / \mathrm{L}$ ammonium acetate, and $1 \mathrm{mmol} / \mathrm{L}$ ascorbic acid in distilled water) was added at room

Table 1 Primer Sequences for Quantitative Real-time PCR

\begin{tabular}{|c|c|c|}
\hline Gene & Forward sequence & Reverse sequence \\
\hline \multicolumn{3}{|l|}{ Human } \\
\hline GAPDH* & $5^{\prime}-\mathrm{TGCACCACCAACTGCTTAGC-3^{ \prime }}$ & $5^{\prime}$-GGCATGGACTGTGGTCATGAG- $3^{\prime}$ \\
\hline$C D 11 b$ & $5^{\prime}-\mathrm{AGAACAACATGCCCAGAACC}-3^{\prime}$ & 5'-GCGGTCCCATATGACAGTCT-3' \\
\hline CD14 & $5^{\prime}$-CGAGGACCTAAAGATAACCGGC- $3^{\prime}$ & $5^{\prime}$-GTTGCAGCTGAGATCGAGCAC- $3^{\prime}$ \\
\hline CD36 & 5'-GCCAGTTGGAGACCTGCTTA-3' & 5'-CAGCGTCCTGGGTTACATTT-3' \\
\hline CCL22 & 5'-TGCCGTGATTACGTCCGTTA-3' & $5^{\prime}$-AAGGTTAGCAACACCACGCC-3' \\
\hline IL1 $\beta$ & 5'-CTCGCCAGTGAAATGATGGCT-3' & 5'-GTCGGAGATTCGTAGCTGGAT-3' \\
\hline$T N F a$ & $5^{\prime}-$ CCAGCTGGAGAAGGGTGAC- $3^{\prime}$ & 5'-AGGCGTTTGGGAAGGTTG-3' \\
\hline \multicolumn{3}{|l|}{ Mouse } \\
\hline Gapdh* & 5'-TGCACCACCAACTGCTTAGC- $3^{\prime}$ & $5^{\prime}$-GGCATGGACTGTGGTCATGAG-3' \\
\hline $\operatorname{Tnf}$ & $5^{\prime}$-GAACTGGCAGAAGAGGCACT-3' & $5^{\prime}$-AGGGTCTGGGCCATAGAACT-3' \\
\hline
\end{tabular}

*Housekeeping genes. 
temperature for 30 minutes. Collected supernatant was read at $570 \mathrm{~nm}$, and cellular iron concentration (in $\mu \mathrm{mol} / \mathrm{L}$ ) was calculated from the FAC standard curve.

\section{Perls Prussian Blue Staining}

Perls Prussian Blue staining was used to visualize iron deposits. HDFs were left on their deposited ECM or removed from their ECM before staining. Plates were fixed (4\% formaldehyde solution) and incubated in a 50:50 solution of $5 \%(w / v)$ potassium ferricyanide (in distilled water; SigmaAldrich) and 5\% (v/v) hydrochloric acid for 10 minutes at room temperature, then rinsed in distilled water ${ }^{20}$ before imaging.

\section{Murine Bone Marrow Macrophage Culture}

Bone marrow was isolated from $\mathrm{NDb}$ and $\mathrm{Db}$ mice with Dulbecco's modified Eagle's medium (Gibco, Thermo Fisher Scientific). Mononuclear phagocytes were differentiated to macrophages with L929 (CCL-1; ATCC) CM, containing macrophage colony-stimulating factor. Macrophages were differentiated for 7 days, serum starved, and then stimulated. Macrophages were left unstimulated (M0) or polarized to M1 or M2 states ${ }^{21}$ in the presence of FAC and left for 24 hours before collecting for quantitative real-time PCR.

\section{Western Blot Analysis}

Western blot analysis was performed on FAC-treated polarized TDMs. Blots were probed with anti-mouse ferritin (clone B-12; Santa Cruz Biotechnology) at 1 $\mu \mathrm{g} / \mathrm{mL}$ concentration for 1 hour at room temperature and detected with SuperSignal West Pico PLUS Chemiluminescent Substrate (Thermo Fisher Scientific).

\section{Transwell Co-Culture}

THP-1 cells were differentiated and polarized, as described above, but in Transwell inserts $(0.4 \mu \mathrm{m}$; Corning, Flintshire, UK). After polarization, inserts were washed in Dulbecco's phosphate-buffered saline, to remove exogenous cytokines and FAC, and transferred to plates seeded with primary HDFs. THP-1 cell-containing inserts were replaced with fresh inserts after 3 days of culture. On day 6, Transwell inserts were removed and HDFs were fixed and stained using anti-mouse collagen type I primary antibody at $1 \mu \mathrm{g} / \mathrm{mL}$ concentration (clone 3G3; Santa Cruz Biotechnology). Images were taken via confocal microscopy (above) and analysis of collagen production performed in ImageJ version 1.8.0. Quantitative real-time PCR was also performed on HDFs after Transwell co-culture.

\section{Proteome Profiler Chemokine Arrays}

TDMs were polarized in the presence of FAC for 24 hours, washed extensively, and then given fresh RPMI 1640 media with no cytokines for 2 days. This medium was collected and sterile filtered before performing Proteome Profiler chemokine arrays (Biotechne, Oxford, UK), as per manufacturer's instructions.

\section{Human ex Vivo Wounding}

Human skin was collected, as described above, and defatted before washing in 2\% (v/v) antibiotic-antimycotic solution (Thermo Fisher Scientific). Partial-thickness wounds were generated (2-mm diameter) in the center of 6-mm-diameter skin explants using sterile biopsy punches (Stiefel Laboratories, Research Triangle Park, NC). Wounds were cultured in Petri dishes on a stack of two absorbent pads and a nitrocellulose 0.22- $\mu \mathrm{m}$ membrane using Dulbecco's modified Eagle's medium with $10 \%$ fetal bovine serum and $1 \%$ antibioticantimycotic solution. Wounds were treated with a vehicle ( $1 \%$ bovine serum albumin plus $0.002 \%$ dimethyl sulfoxide), 100 $\mathrm{nmol} / \mathrm{L}$ CCR 4 antagonist (C 021 dihydrochloride), $50 \mathrm{ng} / \mathrm{mL}$ CCL17, and/or $50 \mathrm{ng} / \mathrm{mL}$ CCL22 (R\&D Systems, Oxford, UK), applied topically every 2 days. Wounds were collected in $4 \%$ neutral-buffered formalin 3 days after wounding for histologic analysis.

\section{Statistical Analysis}

Means \pm SEM were used to present all data. One- and twoway analyses of variance were performed where applicable, with appropriate post-hoc analyses. Significance was deduced at $P<0.05$. Statistical analysis was performed in GraphPad Prism version 7.0 (GraphPad Software, San Diego, CA).

\section{Results}

Macrophages Accumulate Iron across a Murine Healing Time Course

We previously demonstrated the temporal accumulation of iron in wound repair. ${ }^{18}$ Herein, the cellular nature of this accumulated iron (measured as bioavailable ferritin) was assessed across a wound healing time course. Total ferritin was significantly increased on day $3(P<0.001)$ and day 7 $(P<0.001)$ after injury (Figure $1, \mathrm{~A}-\mathrm{C})$, with a large proportion of iron sequestration occurring in macrophages $(P<0.001)$ (Figure 1D) and, to a lesser extent, $\alpha$-smooth muscle actin-positive fibroblasts $(P<0.05)$ (Figure 1E). Of note, wound macrophage phenotype changes significantly over this time frame; nitric oxide synthase 2-positive (proinflammatory) macrophages peaked at day 3 after injury, whereas arginase-1-positive (anti-inflammatory) macrophages dominated at day 7 after injury (Supplemental Figure S1). Hence, wound iron preferentially accumulated 
in macrophages, predominately correlating with tissue infiltration of prohealing (M2) macrophages.

Impaired Macrophage Iron Accumulation in Diabetic Wounds Correlates with Altered Macrophage Polarization

In both $\mathrm{Db}$ and $\mathrm{NDb}$ mouse wounds, ferritin increased at day $3(P<0.01$ and $P<0.05)$ and day $7(P<0.001$ and $P<0.01$ ) after injury, respectively (Figure 2, A and B). However, the absolute ferritin level was reduced in $\mathrm{Db}$ mice at all time points examined, significant at day 7 after injury $(P<0.05)$. Interestingly, although the proportion of wound tissue ferritin-positive macrophages was approximately equal between $\mathrm{Db}$ and $\mathrm{NDb}$ mice day 3 wounds, there was a clear reduction in $\mathrm{Db}$ macrophage iron sequestration at day 7 after injury (Figure 2C). This reduced proportion of ferritin-positive macrophages in day $7 \mathrm{Db}$ wounds was even more meaningful given that total macrophages (Figure 2E) and nitric oxide synthase 2-positive cells (M1 macrophage marker) (Figure 2F) were both increased in $\mathrm{Db}$ mouse wounds. By contrast, wound arginase-1-positive cells (M2 macrophage marker) were reduced in day $7 \mathrm{Db}$ versus $\mathrm{NDb}$ wounds $(P<0.05)$ (Figure 2, D and $\mathrm{G})$, mirroring ferritinpositive macrophages (Figure 2C). Collectively, these data reveal altered macrophage iron sequestration and polarization in $\mathrm{Db}$ wounds.

\section{Endogenous Iron or Iron-Rich Extracellular Matrix Promotes Macrophage Differentiation}

The tissue microenvironment drives monocyte to macrophage differentiation. ${ }^{5} \mathrm{We}$, thus, investigated the influence of exogenous iron on macrophage differentiation in THP-1 cells in vitro. Herein, iron $(100 \mu \mathrm{mol} / \mathrm{L}$ FAC $)$ administration significantly potentiated THP-1 monocyte differentiation in response to PMA treatment, marked by enhanced expression of the differentiation markers $C D 11 b(P<0.001), C D 14$ $(P<0.001), C D 36$, and CD54 $(P<0.001)$ (Figure 3, A-D). FAC also increased cellular adhesion, a phenotypic marker of differentiation $(P<0.001)$ (Figure 3E) and significantly induced cellular matrix metalloproteinase 9 activity $(P<0.001)$ (Figure $3, \mathrm{~F}$ and $\mathrm{G})$.
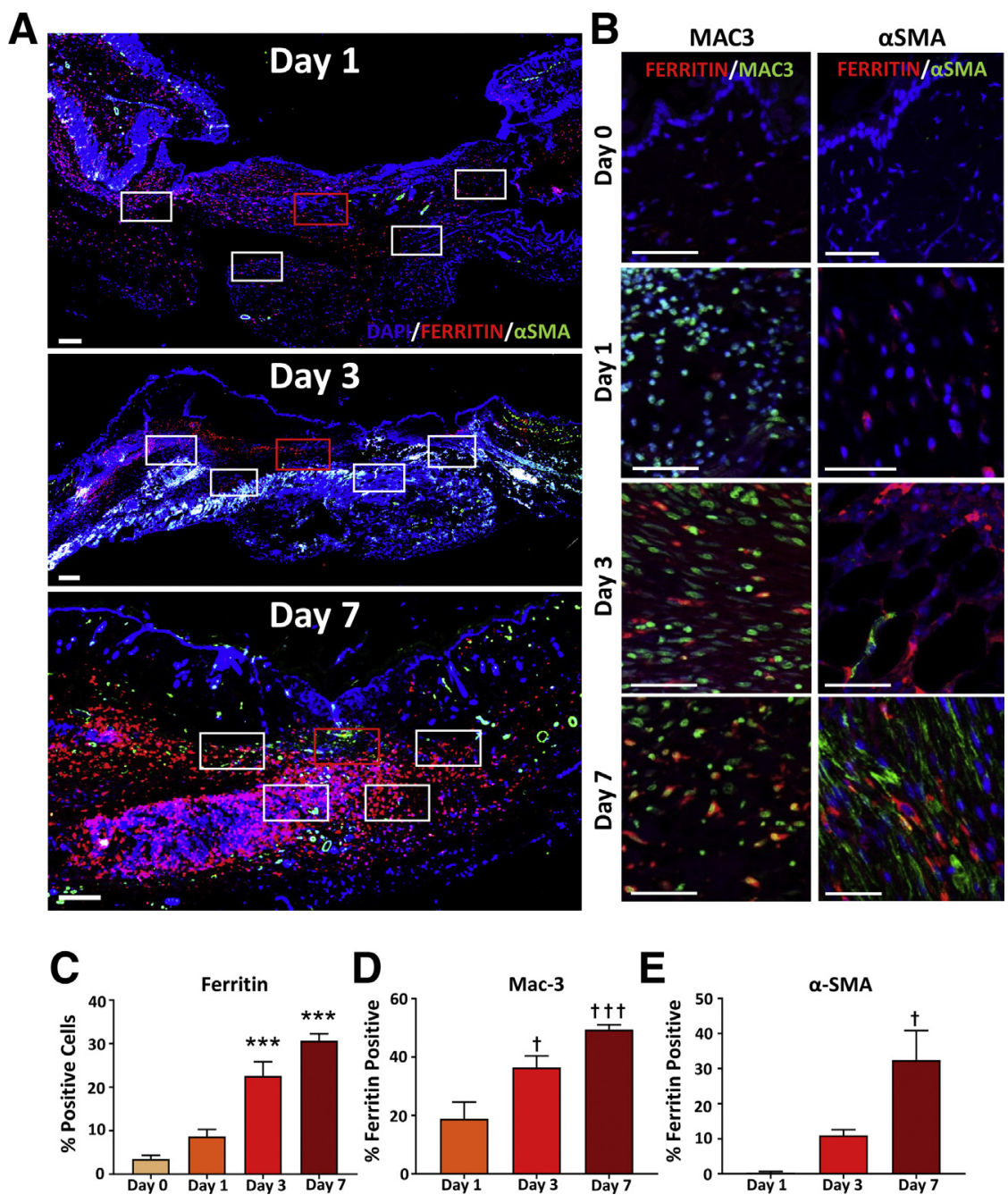

Figure 1 Ferritin levels increase in a temporal manner throughout wound healing, and macrophages are key in time-dependent iron sequestration. A: Full wound images demonstrating ferritin (red) and $\alpha$-smooth muscle actin ( $\alpha$-SMA) (green) staining. DAPI indicates blue nuclei. Five regions in the granulation tissue were analyzed (boxed areas). B: Representative images from red boxed areas in A. C-E: Ferritin-positive cells (C), ferritinpositive macrophages (Mac-3; D), and ferritinpositive fibroblasts $(\alpha$-SMA; E) increase throughout healing. Significance versus day 0 (C) and day 1 (D and $\mathbf{E})$. One-way analysis of variance with Tukey post-hoc analysis was performed. Data are expressed as means + SEM (C-E). $n=4$ to 5 mice per group $(\mathbf{A}-\mathbf{E}) .{ }^{* * *} P<0.001$ versus day 0 ; ${ }^{\dagger} P<0.05,{ }^{\dagger \dagger} P<0.001$ versus day 3 . Scale bars: $100 \mu \mathrm{m}$ (A); $50 \mu \mathrm{m}$ (B). 

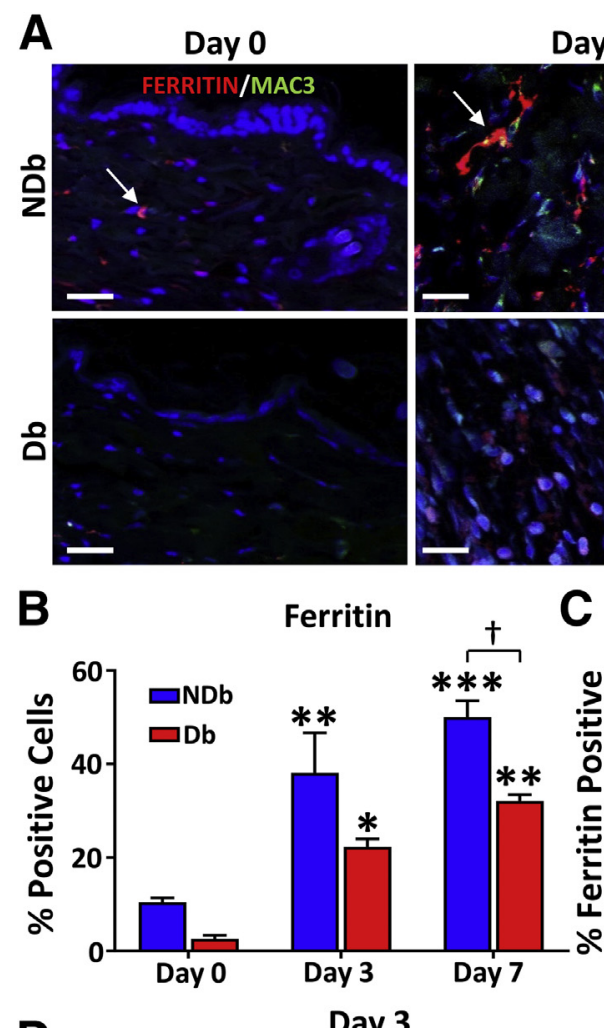

D

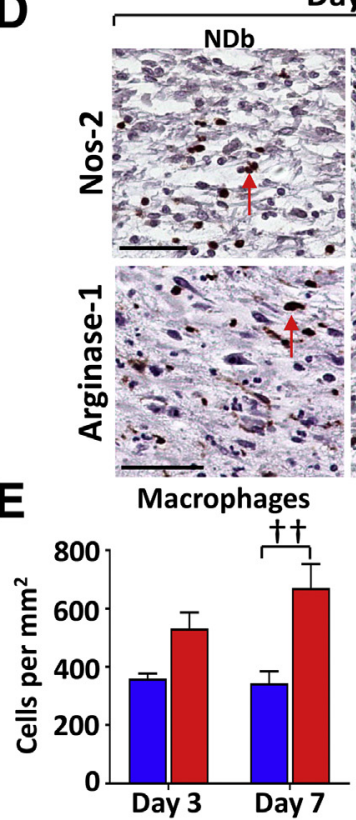

$\widetilde{\mathbf{F}}$
Day 3

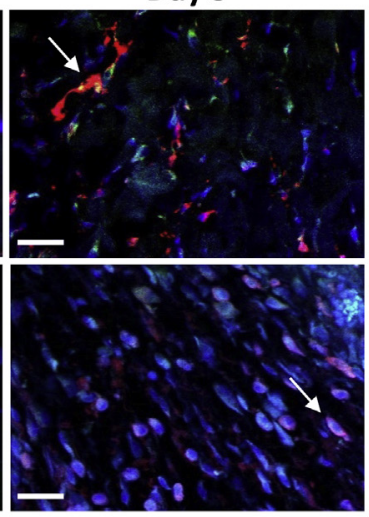

C

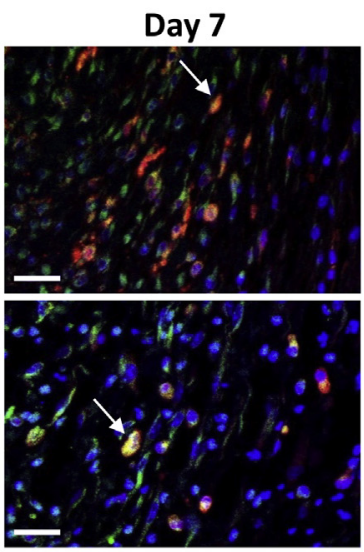

Macrophages

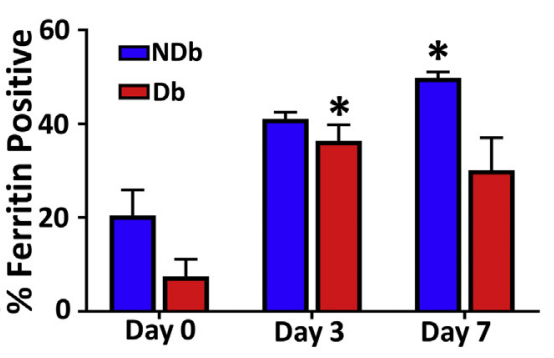

Day 7

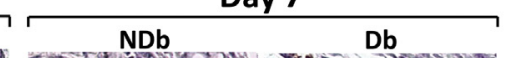

NDb Db
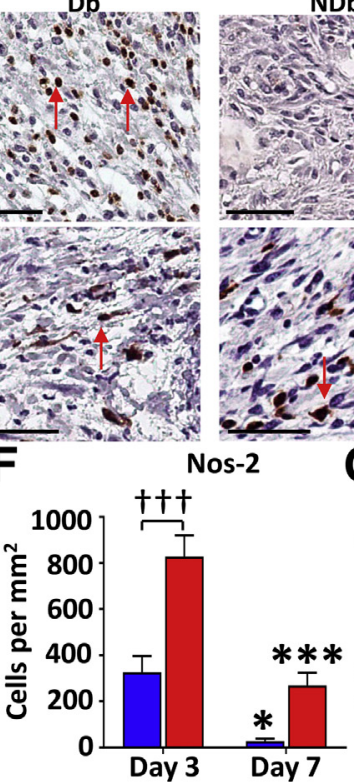

G

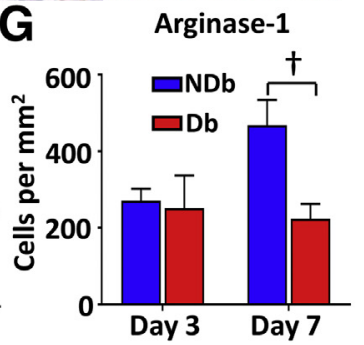

Figure 2 Diabetic $(\mathrm{Db})$ wound macrophages show impaired ferritin sequestration and altered polarization. A: Nondiabetic (NDb) and Db wounds stained for macrophages (Mac-3; green) and ferritin (red). DAPI indicates blue nuclei. Arrows denote ferritin-positive macrophages. B and C: Quantification of total ferritin-positive cells (B) and ferritin-positive macrophages (C). D: Immunoperoxidase staining for nitric oxide synthase 2 (Nos-2) and arginase-1. Arrows denote positive staining. E-G: Quantification of wound macrophages (E), Nos-2-positive macrophages (F) and arginase-1-positive macrophages (G). B, C, and E-G: $P$ values (asterisks) are given versus day 0 (B and $\mathbf{C}$ ) or day $3(\mathbf{E}-\mathbf{G})$. Two-way analysis of variance with Tukey post-hoc analysis was performed. Data are expressed as means + SEM (B, C, and $\mathbf{E}-\mathbf{G}) . n=3$ to 5 mice per group $(\mathbf{A}-\mathbf{G}) .{ }^{*} P<0.05,{ }^{*} P<0.01$, and $* * * P<0.001$ versus day $0 ;{ }^{\dagger} P<0.05,{ }^{\dagger \dagger} P<0.01$, and ${ }^{\dagger \dagger \dagger} P<0.001$ versus day 3 . Scale bars $=50 \mu \mathrm{m}$ (A and D).
In the wound environment, macrophages will be exposed to iron deposited in the ECM; it was, therefore, tested how ECM derived from FAC-treated HDFs influenced macrophage differentiation. HDFs treated with FAC deposited an extensive ECM (Figure $3 \mathrm{H}$ ) rich in iron, as demonstrated by both Perls Prussian Blue staining (Figure 3I) and ferrozine assay (Figure 3J). THP-1 monocytes were seeded onto this denuded ECM and stimulated with PMA to induce differentiation. Exposure to the iron-rich ECM significantly increased expression of the differentiation markers $C D 11 b$
$(P<0.001)$, CD14 $(P<0.05)$, CD36 $(P<0.001)$, and CD54 (Figure 3, K-N). Clearly, both exogenous iron treatment and exposure to iron-rich ECM stimulate macrophage differentiation.

Iron Drives Cytokine-Stimulated Macrophages toward a Prohealing Phenotype

FAC treatment $(100 \mu \mathrm{mol} / \mathrm{L})$ led to a significant decrease in the M1 markers IL-1 $\beta(P<0.01)$ (Figure $4 \mathrm{~A})$ and tumor 

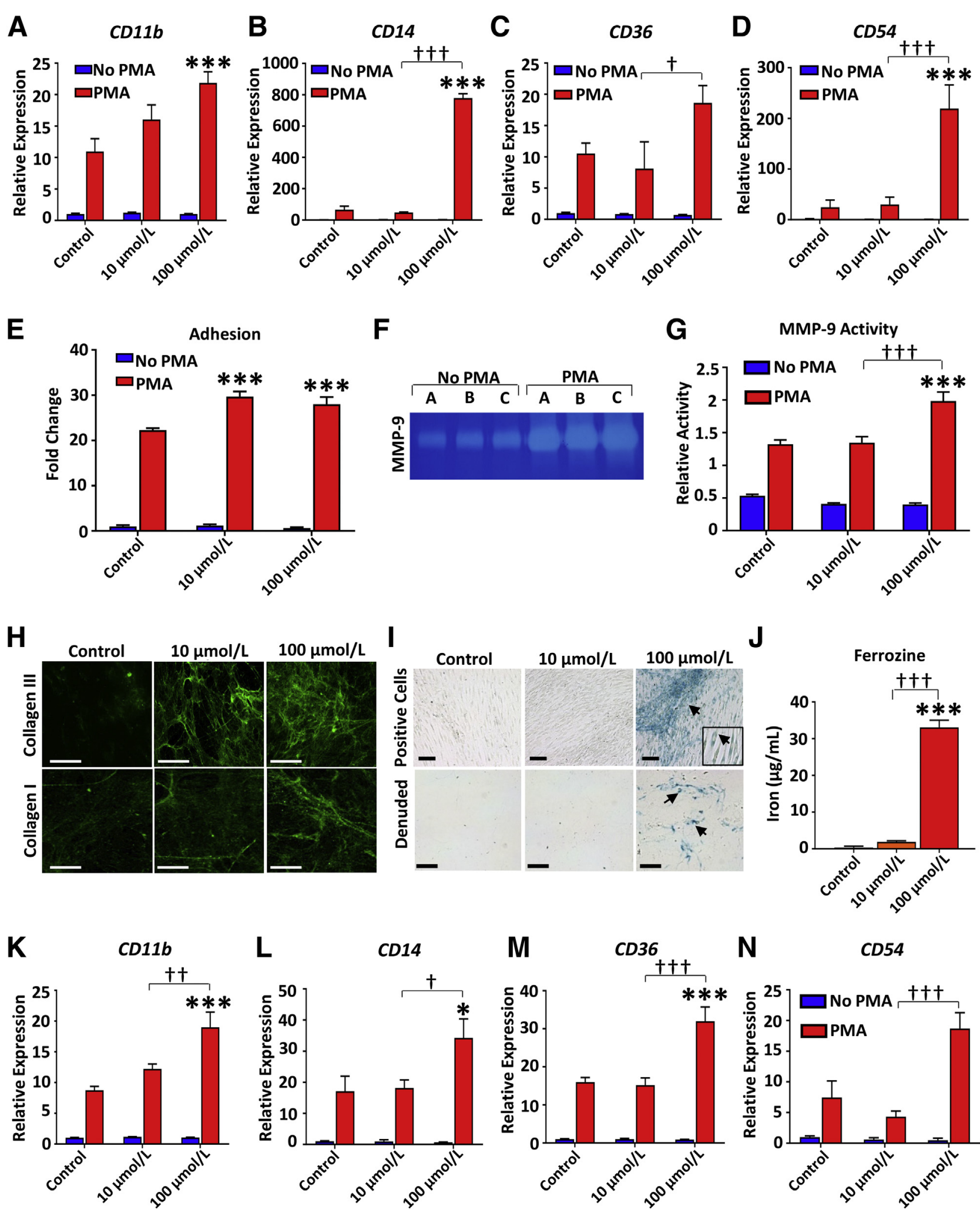

Figure 3 A high iron environment enhances phorbol 12-myristate 13-acetate (PMA)-induced differentiation in THP-1 macrophages (TDMs). TDMs were treated with ferric ammonium citrate (FAC) at the same time as PMA stimulation for differentiation. A-D: CD11b (A), CD14 (B), CD36 (C), and CD54 (D) expression (quantitative real-time PCR, normalized to no PMA, no FAC group). E-G: FAC treatment promotes adhesion (E) and matrix metalloproteinase 9 activity (F and $\mathbf{G}$ ). H-J: Human dermal fibroblasts (HDFs) deposit extracellular matrix (ECM) high in collagens (green; $\mathbf{H}$ ) and rich in iron, shown by Perls Prussian Blue staining (I) and ferrozine assay (J). Arrows indicate blue iron deposits. I: Inset: intracellular iron sequestration. $\mathbf{K}-\mathbf{N}$ : TDMs seeded onto FAC-treated HDF ECM display increased expression (quantitative real-time PCR) of differentiation markers. Two-way analysis of variance with Tukey post-hoc analysis was performed. Data are expressed as means + SEM $(\mathbf{A}-\mathbf{E}, \mathbf{G}$, and $\mathbf{J}-\mathbf{N}) . n=3$ donors $/ 3$ independent experiments $(\mathbf{A}-\mathbf{N}) .{ }^{*} P<0.05$, ${ }^{* * *} P<0.001$ versus control, no FAC group;

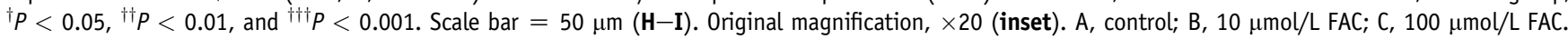




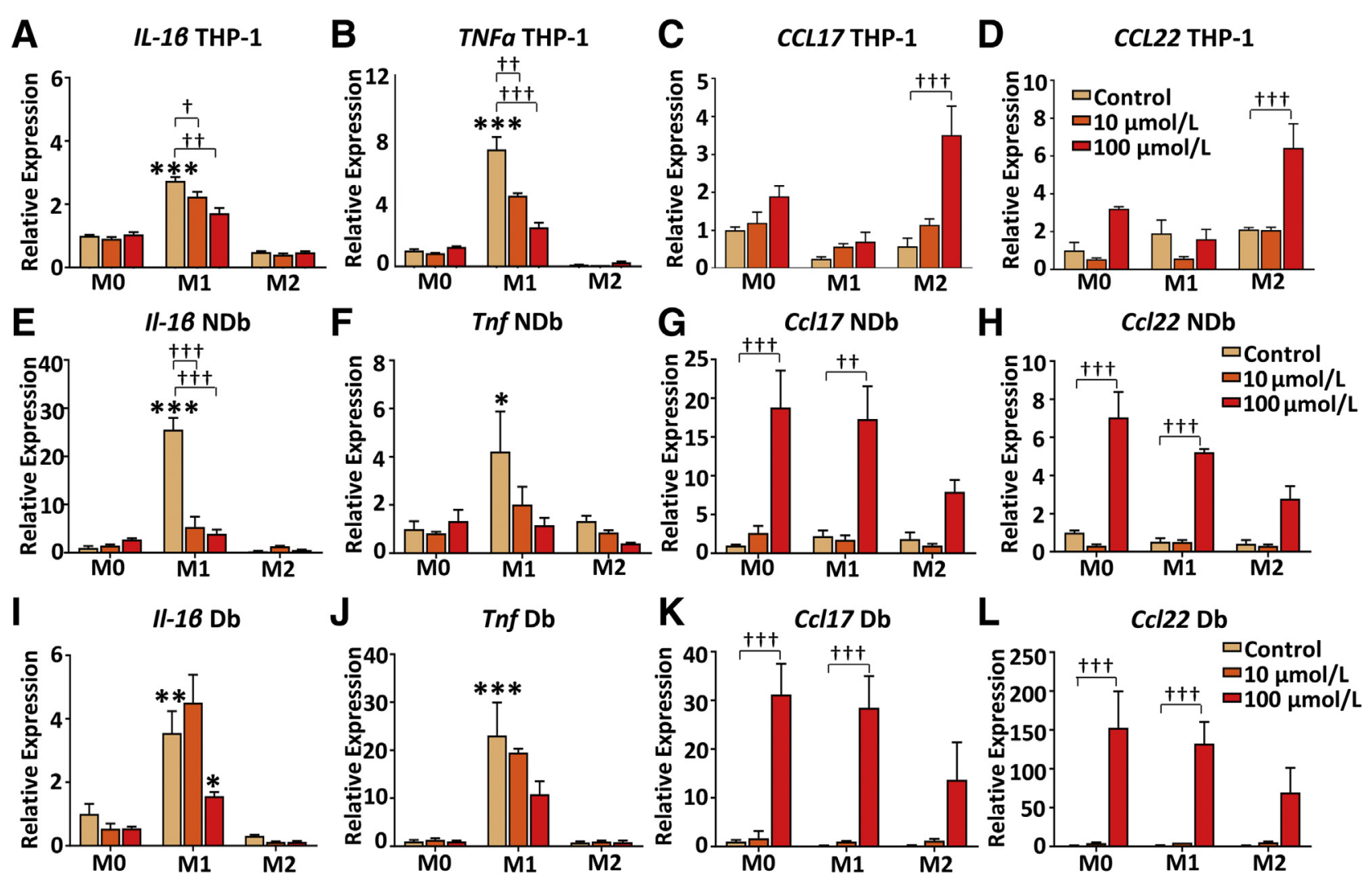

Figure 4 Iron skews macrophages toward a prohealing state. THP-1 macrophages were treated with ferric ammonium citrate (FAC) for 24 hours before quantitative real-time PCR for M1 [IL-1 $\beta$ and tumor necrosis factor (TNF)- $\alpha ; \mathbf{A}$ and B] and M2 (CCL17 and CCL22; C and D) markers. Nondiabetic (NDb) and diabetic (Db) macrophage expression of $\mathrm{Il}-1 \beta(\mathbf{E}$ and $\mathbf{I}), \operatorname{Infa}(\mathbf{F}$ and $\mathbf{J}), \mathrm{Ccl17}(\mathbf{G}$ and $\mathbf{K})$, and $\mathrm{Ccl} 22$ ( $\mathbf{H}$ and $\mathbf{L})$. Normalized to M0, no FAC group. Two-way analysis of variance with Tukey post-hoc analysis was performed. Data are expressed as means + SEM $(\mathbf{A}-\mathbf{L}) . n=3$ donors $/ 3$ independent experiments (A-L). ${ }^{*} P<0.05,{ }^{* *} P<0.01$, and ${ }^{* *} P<<0.001$ versus M0 group; ${ }^{\dagger} P<0.05,{ }^{\dagger \dagger} P<0.01$, and ${ }^{\dagger \dagger \dagger} P<0.001$.

necrosis factor- $\alpha(P<0.001)$ (Figure 4B) in M1-stimulated TDMs, whereas the expression of the M2 markers CCL17 $(P<0.001)$ (Figure 4C) and CCL22 $(P<0.001)$ (Figure 4D) was elevated in M2-stimulated TDMs after 100 $\mu \mathrm{mol} / \mathrm{L}$ FAC. Similar effects were observed in NDb murine bone marrow-derived macrophages (BMDMs), where $I l-1 \beta$ expression was reduced at both FAC concentrations $(P<0.001)$ (Figure 4E). Db BMDMs were less responsive to iron, with $I l-1 \beta$ expression only reduced by $100 \mu \mathrm{mol} / \mathrm{L}$ FAC treatment $(P<0.05)$ (Figure $4 \mathrm{I})$ whereas FAC treatment modestly reduced Tnf in NDb and Db BMDMs (Figure 4, J and L). Intriguingly, $100 \mu \mathrm{mol} / \mathrm{L}$ FAC treatment elevated $C c l 17$ and $C c l 22$ in M0, M1, and M2 polarized BMDMs from $\mathrm{NDb}$ and $\mathrm{Db}$ mice (Figure 4, G, H, K, and L). Together, these data reveal that iron skews cytokine profiles in both TDMs and BMDMs.

\section{Iron-Loaded M2 Macrophages Secrete Factors that Stimulate Extracellular Matrix Production}

TDMs treated with $100 \mu \mathrm{mol} / \mathrm{L}$ FAC showed significantly greater ferritin storage in M0 $(P<0.001)$ and M2 $(P<0.001)$ states (Figure 5, A and B) by immunohistochemistry, and in all three activation states via Western blot analysis $(P<0.01$ to $P<0.001)$ (Figure 5 C). For both techniques, the highest levels of ferritin were observed in $100 \mu \mathrm{mol} / \mathrm{L}$ FAC-treated M2-polarized TDMs. As macrophages play a key role in ECM remodeling, ${ }^{28}$ a Transwell co-culture approach was used to test the paracrine effects of FAC-stimulated TDMs on HDF ECM production. Transwell co-culture of M2-stimulated FAC-treated TDMs resulted in up-regulation of COL1AI $(P<0.05)$ (Figure 5D), COL1A2 $(P<0.001)$ (Figure 5E), and COL3A1 $(P<0.05)$ (Figure 5F) expression in HDFs. Collagen I protein levels were also highest in HDFs co-cultured with M2-stimulated, FAC-treated TDMs $(P<0.001)$ (Figure 5, G and $\mathrm{H})$. Finally, $\mathrm{CM}$ from irontreated M2-polarized TDMs was profiled using chemokine arrays to characterize secreted factors of interest (Figure 5I). Herein, CM from M2-stimulated TDMs treated with 100 $\mu \mathrm{mol} / \mathrm{L}$ FAC displayed higher levels of multiple chemokines, including CCL17 and CCL22.

CCL17 and CCL22 Promote Healing and ECM Deposition in Human ex Vivo Wound Repair

To test the biological significance of TDM-derived chemokines, an ex vivo wound model was used. Given that the 
A

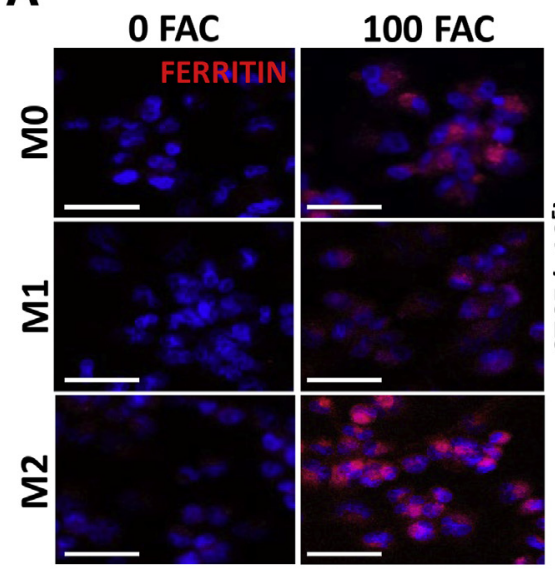

B

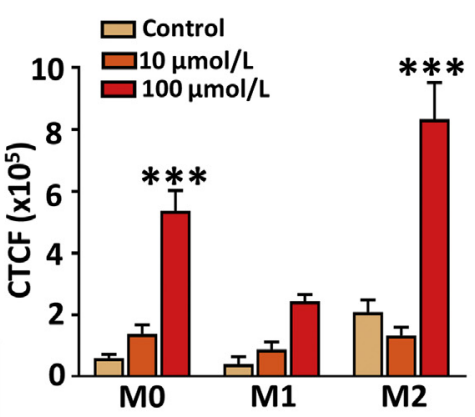

C

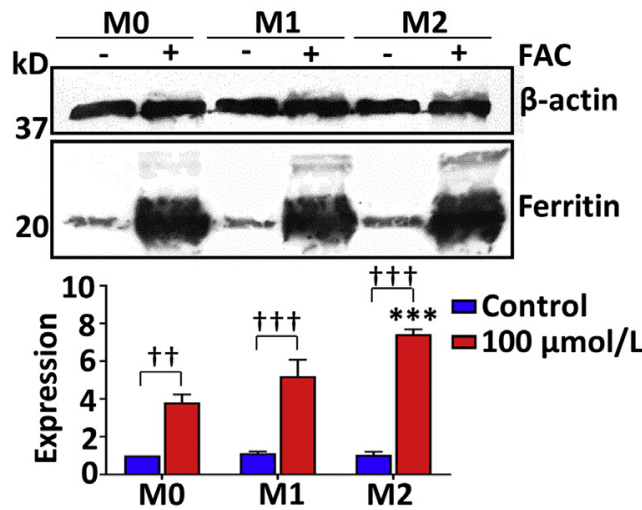

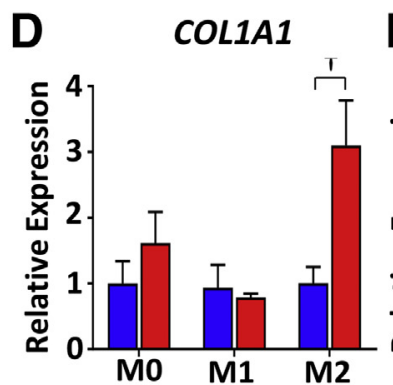

E COL1A2

$\mathbf{F}$
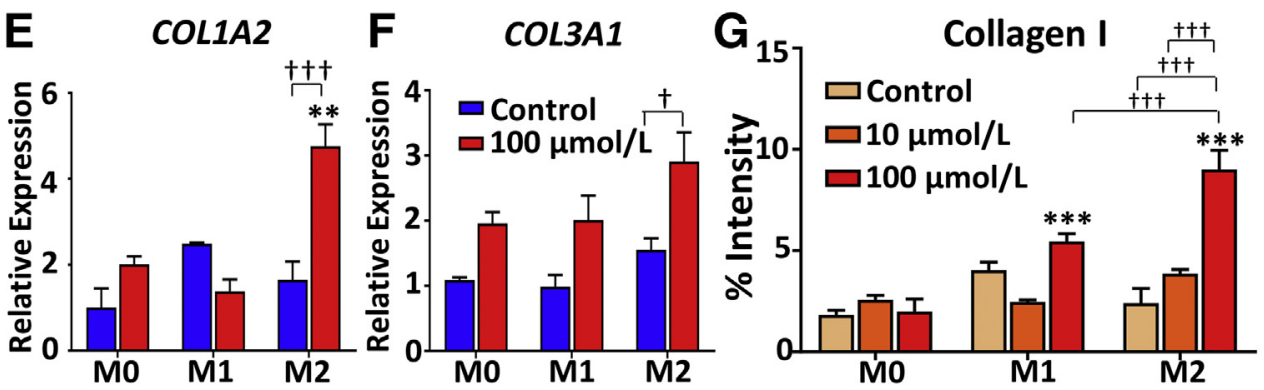

H

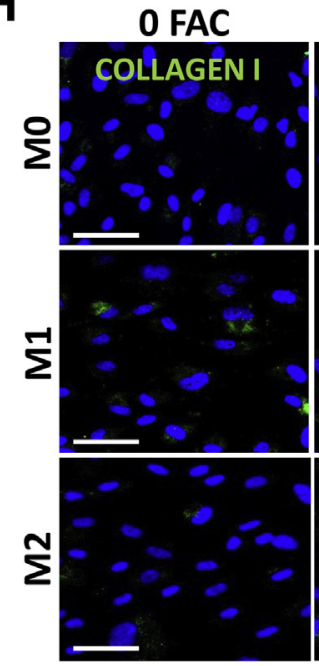

10 FAC
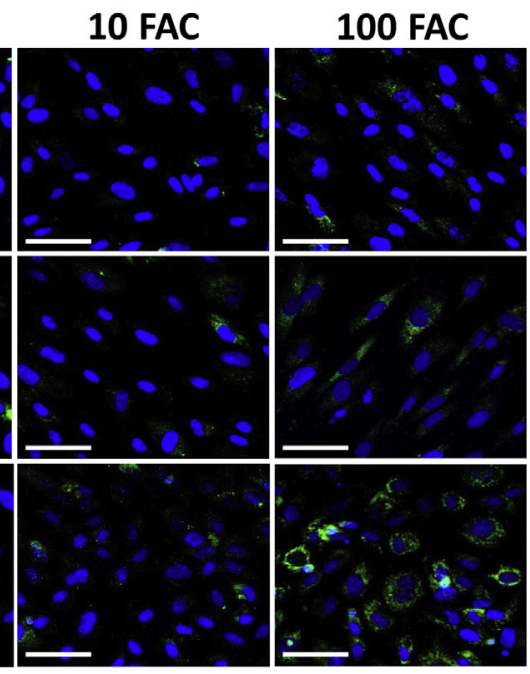

I

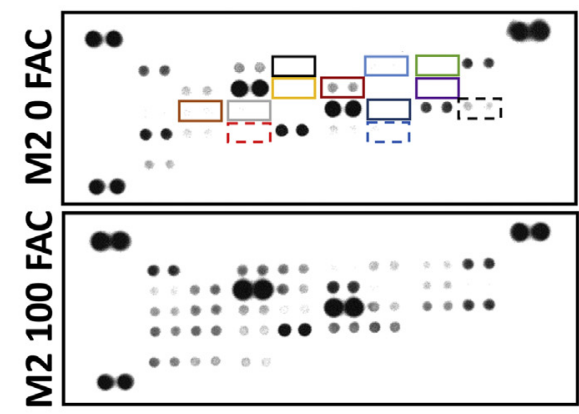

$\square$ Chimerin $\square$ IL-16 $\square$ CCL22

$\begin{array}{lll}\text { CCL26 } & \square \text { CXCL10 } \square \text { Midkine } \\ \text { CX3CL1 } & \square C L 1 & \text { CXCL4 }\end{array}$

Figure 5 Iron-loaded THP-1 macrophages (TDMs) secrete factors that stimulate extracellular matrix production in human dermal fibroblasts (HDFs). A-C: Ferric ammonium citrate (FAC)-treated TDMs show increased ferritin expression via immunocytochemistry (A and B) and Western blot analysis (C). + FAC indicates $100 \mu \mathrm{mol} / \mathrm{L}$. D-H: Transwell co-culture of these FAC pretreated TDMs with HDFs leads to up-regulation of COL1A1 (D), COL1A2 (E), and COL3A1 (F) via quantitative real-time PCR, and increased collagen I production via immunofluorescence $(\mathbf{G}$ and $\mathbf{H})$, in HDFs. I: Antibody array demonstrates increased production of chemokines in FAC-treated THP-1-conditioned media. Two-way analysis of variance with Tukey post-hoc analysis was performed. Data are expressed + SEM (B-G). $n=3$ donors $/ 3$ independent experiments $(\mathbf{A}-\mathbf{I}) .{ }^{* *} P<0.01,{ }^{* *} P<0.001$ versus $M 0$ group; ${ }^{\dagger} P<0.05,{ }^{\dagger \dagger} P<0.01$, and $t_{\dagger \dagger} P<0.001$. Scale bars $=50 \mu \mathrm{m}(\mathbf{A}$ and $\mathbf{H})$. CTCF, corrected total cell fluorescence.

CCL17-CCR4 receptor axis has previously been suggested to be important for healing, ${ }^{29}$ CCL17, CCL22, and an antagonist to their common receptor, CCR $4,{ }^{30}$ were applied topically to human ex vivo wounds. Wound closure (reepithelialisation) was significantly increased after treatment with CCL17 $(P<0.01)$ or CCL22 $(P<0.05)$ in isolation and by a combination of both ligands $(P<0.001)$ (Figure 6 ,
A and B). Although the CCR4 antagonist (C 021 dihydrochloride) had no direct effect on wound closure, it significantly blocked the healing promoting effect of CCL17 $(P<0.05)$ and a combination of both ligands $(P<0.01)$. Finally, CCL17 and CCL22 effects on ECM production were evaluated. Collagen I and collagen III contents were assessed from three regions close to the wound surface 


\section{A}
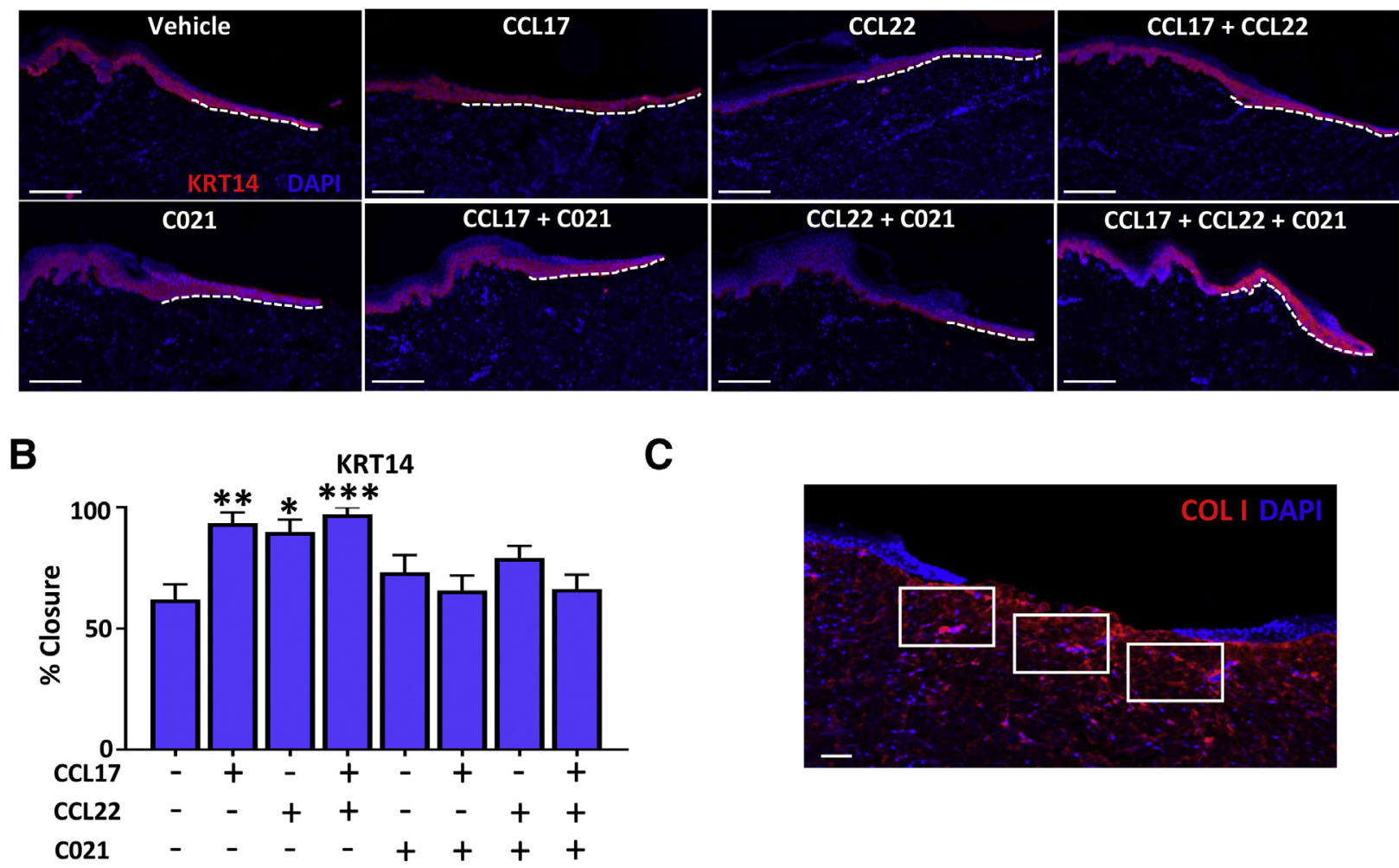

C

D

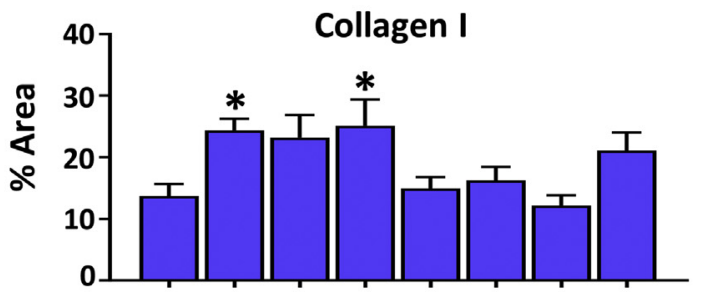

Collagen III

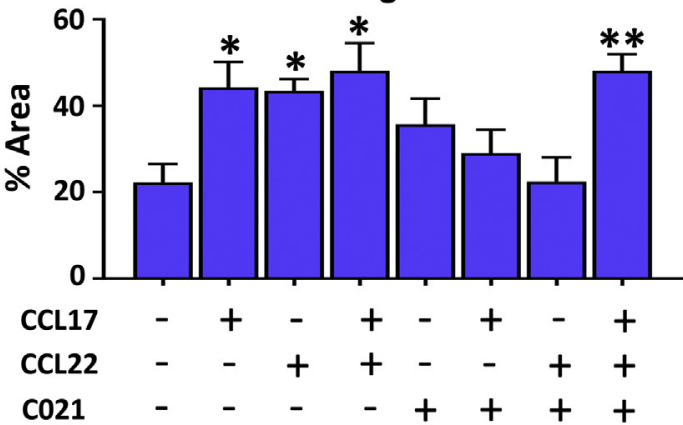

$\mathbf{E}$

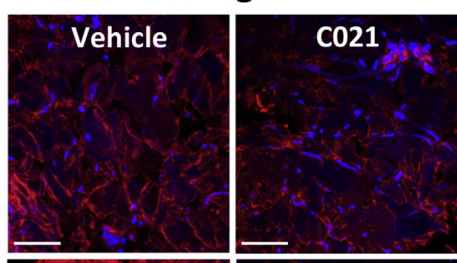

CCL17

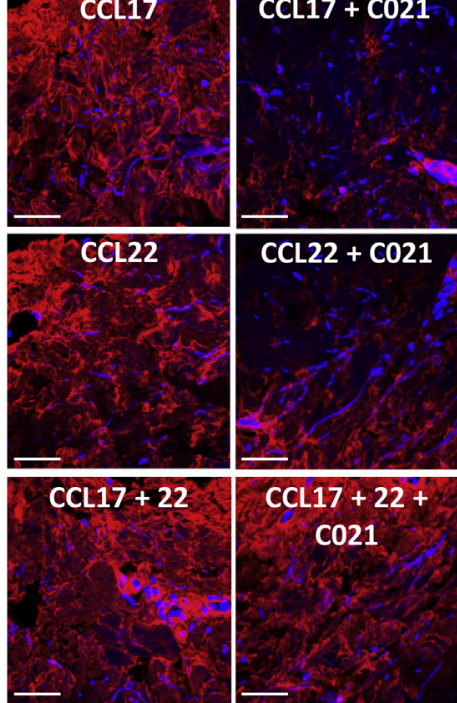

F Collagen III

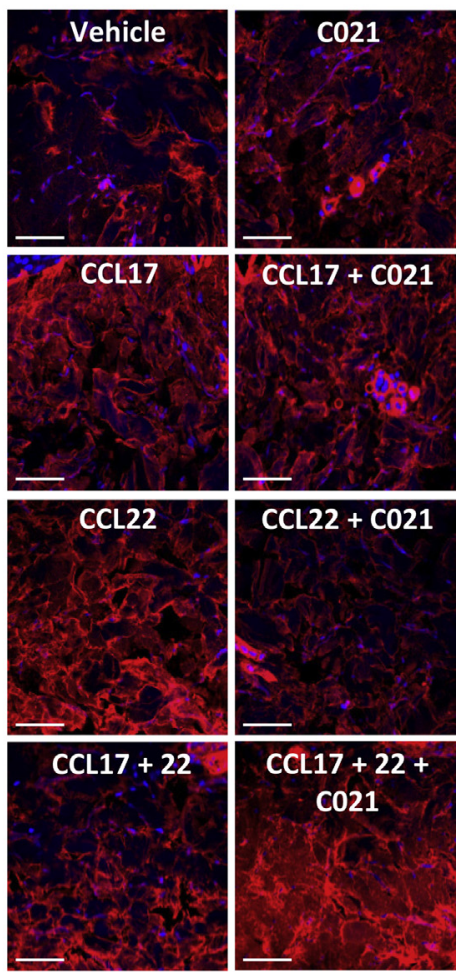

Figure 6 CCL17 and CCL22 promote healing during ex vivo human wound repair. A and B: Keratin 14 staining (KRT14; A) and quantification of reepithelialization (\% closure; B). Dotted lines demonstrate reepithelialization. C: Representative image of collagen (COL) immunofluorescence quantification from three regions of each section (boxed areas). D-F: Quantification of collagen I and collagen III immunofluorescence (D) and representative immunofluorescence images (E and F). One-way analysis of variance with Tukey post-hoc analysis was performed. Data are expressed as means + SEM $(\mathbf{B}$ and $\mathbf{D}) . n=3$ donors $(\mathbf{A}-\mathbf{F})$. ${ }^{\star} P<0.05,{ }^{*} P<0.01$, and ${ }^{* *} P<0.001$ versus vehicle group. Scale bars $=50 \mu \mathrm{m}$ (A, C, E, and F). C021, CCR4 antagonist (C 021 dihydrochloride). 
(Figure 6C). Collagen I was significantly increased after treatment with CCL17 $(P<0.05)$ or a combination of both ligands $(P<0.05)$, an effect that was again reversed by cotreatment with the antagonist, C 021 dihydrochloride (Figure 6, D and E). Collagen III was significantly elevated after treatment with both ligands alone and in combination $(P<0.05)$, which was again blocked by the receptor antagonist, C 021 dihydrochloride (Figure 6, D and F). Together, these data reveal a novel, previously unappreciated role for the macrophage-derived chemokines, CCL17 and CCL22, in promoting human wound reepithelialization and matrix deposition.

\section{Discussion}

Macrophages are closely associated with the biological availability of iron. In addition to modulating heme storage and erythrocyte recycling, ${ }^{16}$ they engulf damaged erythrocytes in the wound bed. ${ }^{20}$ Wound tissue macrophages present with diverse phenotypes, ${ }^{31}$ yet how macrophage behavior is influenced by the complex wound microenvironment remains far from clear. Herein, we present new experimental findings linking iron modulation of macrophage behavior and the remodeling phase of wound repair.

We previously revealed temporal accumulation of iron in late-stage wound repair. ${ }^{18}$ Indeed, this wound iron sequestration correlates with a macrophage switch to an antiinflammatory (M2) phenotype, ${ }^{32}$ which is impaired in $\mathrm{Db}$ pathologic healing. ${ }^{33}$ Circulating monocytes differentiate into macrophages in response to environmental cues, potentially including iron. In fact, iron deficiency reduces CD14 expression in blood monocyte-derived macrophage $^{34}$ and heme iron mediates SPI-C-induced macrophage differentiation. ${ }^{15}$ In concurrence, the present study revealed that exposure to high iron increased macrophage differentiation, but also significantly extended this observation to demonstrate a role for iron in modulating macrophage polarization and chemokine secretion.

Macrophages are able to perform versatile functions with high plasticity to allow effective repair. Functionally, CD11b-specific ${ }^{35}$ and arginase-specific ${ }^{12}$ ablation leads to delayed healing and excessive inflammation. This illustrates not only the importance of macrophage presence, but also effective temporal modulation of macrophage phenotype in wound healing. Although not extensively characterized, the late-stage M2 switch may be driven by degradation of M1 receptors $^{36}$ and activation of STAT6 and peroxisome proliferator-activated receptor- $\gamma \cdot{ }^{37}$ Herein, we show that iron may also critically promote alternative activation by reducing the expression of $I l-1 \beta$ and Tnf- $\alpha$ and upregulating the expression of $C C L 17$ and $C C L 22$.

The effect of iron on macrophage polarization and phenotype appears to be context dependent. Heme uptake by macrophages in the damaged spinal cord promotes M1driven inflammation in mice, ${ }^{38}$ whereas iron sequestration induced an M1 phenotype in raw246.7 macrophages. ${ }^{39}$ It has even been suggested that sustained, iron-induced, proinflammatory macrophages may contribute to delayed healing by triggering fibroblast senescence. ${ }^{40,41}$ On the contrary, in atherosclerotic plaques, hemoglobinhaptoglobin complexes shifted macrophages toward an M2 state both in vivo (higher CD163) and in vitro (increased IL-10 and IL-1RA ${ }^{42}$ ). Likewise, high iron up-regulated M2 marker expression, and reduced proinflammatory cytokines, in lipopolysaccharide-stimulated macrophages. ${ }^{43}$

M2 macrophages release a myriad of factors (eg, transforming growth factor- $\beta 1$ ) to promote reepithelialization, fibroplasia, fibroblast differentiation, ${ }^{13}$ and ECM remodeling. ${ }^{29}$ In the current study, it is shown, for the first time, that iron treatment of macrophages significantly potentiated the release of a range of chemokines, which promoted collagen production in HDFs. M2 macrophage $\mathrm{CM}$ is known to stimulate collagen 1 and collagen 3 production in fibroblasts in vitro, ${ }^{44}$ but direct application of M2-stimulated BMDMs did not alter $\mathrm{Db}$ healing in vivo. ${ }^{45}$ These new data suggest that iron indirectly stimulates HDF ECM deposition via modulation of macrophage behavior, with potential therapeutic application in pathologic healing.

The iron-treated M2 macrophage screen identified the chemokines CCL17 and CCL22. Both CCL17 and CCL22 were shown to directly promote reepithelialization and collagen deposition in a human ex vivo wound repair model. This is in line with previous work in $\mathrm{HaCaTs}^{46}$ fibroblasts, ${ }^{47}$ and fibrosis in vivo. ${ }^{48}$ CCL17 has also been shown to promote wound closure and granulation tissue formation in murine wounds in vivo. ${ }^{49}$ Curiously, Barros et $\mathrm{al}^{50}$ demonstrated improved healing in a CCR $4^{-1-}$ type 1 diabetes mellitus model and in wild-type type 1 diabetes mellitus mice treated with anti-CCL17 and anti-CCL22 antibodies. Thus, future studies investigating macrophage iron processing should be extended to address tissue- and pathology-specific differences.

In summary, these new data demonstrate a key role for iron in modulating macrophage responses to the wound environment. Iron skewed macrophages toward an M2-like state that produced a secretome highly enriched with ECMstimulating factors, including the chemokines, CCL17 and CCL22. It is tempting to speculate that local iron administration, perhaps targeted to the latter stages of wound healing, could have clinical benefit. Further work is now required to explore how wound iron manipulation can be used as a conduit to improve skin healing pathology.

\section{Acknowledgments}

We thank Dr. Barbara Guinn for helpful discussions.

H.N.W. and M.J.H. designed the study; H.N.W., E.R.R., and A.R.S. acquired data; H.N.W., E.R.R., and K.L.B. analyzed the data; H.N.W. prepared the manuscript; H.N.W., E.R.R., K.A.M., and M.J.H. revised the 
manuscript; K.A.M. and M.J.H. supervised the study; all authors read and approved the final manuscript; M.J.H. is the guarantor of this work and, as such, had full access to all the data in the study and takes responsibility for the integrity of the data and the accuracy of the data analysis.

\section{Supplemental Data}

Supplemental material for this article can be found at http://doi.org/10.1016/j.ajpath.2019.07.015.

\section{References}

1. Mantovani A, Sica A, Locati M: Macrophage polarization comes of age. Immunity 2005, 23:344-346

2. Stuart LM: Ezekowitz RAB: Phagocytosis: elegant complexity. Immunity 2005, 22:539-550

3. Zhou D, Huang C, Lin Z, Zhan S, Kong L, Fang C, Li J: Macrophage polarization and function with emphasis on the evolving roles of coordinated regulation of cellular signaling pathways. Cell Signal 2014, 26:192-197

4. Boniakowski AE, Kimball AS, Jacobs BN, Kunkel SL, Gallagher KA: Macrophage-mediated inflammation in normal and diabetic wound healing. J Immunol 2017, 199:17-24

5. Das A, Sinha M, Datta S, Abas M, Chaffee S, Sen CK, Roy S: Monocyte and macrophage plasticity in tissue repair and regeneration. Am J Pathol 2015, 185:2596-2606

6. Ginhoux F, Guilliams M: Tissue-resident macrophage ontogeny and homeostasis. Immunity 2016, 44:439-449

7. Snyder RJ, Lantis J, Kirsner RS, Shah V, Molyneaux M, Carter MJ: Macrophages: a review of their role in wound healing and their therapeutic use. Wound Repair Regen 2016, 24:613-629

8. Mosser DM, Edwards JP: Exploring the full spectrum of macrophage activation. Nat Rev Immunol 2008, 8:958-969

9. Hesketh M, Sahin KB, West ZE, Murray RZ: Macrophage phenotypes regulate scar formation and chronic wound healing. Int J Mol Sci 2017, 18:e1545

10. Korns D, Frasch SC, Fernandez-Boyanapalli R, Henson PM, Bratton DL: Modulation of macrophage efferocytosis in inflammation. Front Immunol 2011, 2:57

11. Eming SA, Wynn TA, Martin P: Inflammation and metabolism in tissue repair and regeneration. Science 2017, 356:1026-1030

12. Campbell L, Saville CR, Murray PJ, Cruickshank SM, Hardman MJ: Local arginase 1 activity is required for cutaneous wound healing. J Invest Dermatol 2013, 133:2461-2470

13. Li J, Chen J, Kirsner R: Pathophysiology of acute wound healing. Clin Dermatol 2007, 25:9-18

14. Sukhbaatar N, Weichhart T: Iron regulation: macrophages in control. Pharmaceuticals 2018, 11:e137

15. Haldar M, Kohyama M, So AY, Kc W, Wu X, Briseño CG, Satpathy AT, Kretzer NM, Arase H, Rajasekaran NS, Wang L, Egawa T, Igarashi K, Baltimore D, Murphy TL, Murphy KM: Hememediated SPI-C induction promotes monocyte differentiation into iron-recycling macrophages. Cell 2014, 156:1223-1234

16. Arezes J, Nemeth E: Hepcidin and iron disorders: new biology and clinical approaches. Int J Lab Hematol 2015, 37:92-98

17. Zhang Z, Zhang F, An P, Guo X, Shen Y, Tao Y, Wu Q, Zhang Y, Yu Y, Ning B, Nie G: Ferroportin1 deficiency in mouse macrophages impairs iron homeostasis and inflammatory responses. Blood 2011, 118:1912-1922

18. Wilkinson HN, Upson SE, Banyard KL, Knight R, Mace KA, Hardman MJ: Reduced iron in diabetic wounds: an oxidative stressdependent role for STEAP3 in extracellular matrix deposition and remodelling. J Invest Dermatol 2019, [Epub ahead of print] doi:10 1016/j.jid.2019.05.014

19. Yeoh-Ellerton S, Stacey MC: Iron and 8-isoprostane levels in acute and chronic wounds. J Invest Dermatol 2003, 121:918-925

20. Sindrilaru A, Peters T, Wieschalka S, Baican C, Baican A, Peter H, Hainzl A, Schatz S, Qi Y, Schlecht A, Weiss JM: An unrestrained proinflammatory M1 macrophage population induced by iron impairs wound healing in humans and mice. J Clin Invest 2011, 121:985-997

21. Wilkinson HN, Clowes C, Banyard KL, Matteuci P, Mace KA, Hardman MJ: Elevated local senescence in diabetic wound healing is linked to pathological repair via CXCR2. J Invest Dermatol 2019, 139:1171-1181

22. Bosshart H, Heinzelmann M: THP-1 cells as a model for human monocytes. Ann Transl Med 2016, 4:e438

23. Michée S, Brignole-Baudouin F, Riancho L, Rostene W, Baudouin C, Labbé A: Effects of benzalkonium chloride on THP-1 differentiated macrophages in vitro. PLoS One 2013, 8:e72459

24. Wang F, Gao Q, Guo S, Cheng J, Sun X, Li Q, Wang T, Zhang Z, Cao W, Tian Y: The sonodynamic effect of curcumin on THP-1 cellderived macrophages. Biomed Res Int 2013, 2013:e737264

25. Chomczynski P, Sacchi N: Single-step method of RNA isolation by acid guanidinium thiocyanate-phenol-chloroform extraction. Anal Biochem 1987, 162:156-159

26. Wilkinson $\mathrm{H}$, Iveson $\mathrm{S}$, Catherall $\mathrm{P}$, Hardman M: A novel silver bioactive glass elicits antimicrobial efficacy against Pseudomonas aeruginosa and Staphylococcus aureus in an ex vivo skin wound biofilm model. Front Microbiol 2018, 9:e1450

27. Riemer J, Hoepken HH, Czerwinska H, Robinson SR, Dringen R: Colorimetric ferrozine-based assay for the quantitation of iron in cultured cells. Anal Biochem 2004, 331:370-375

28. Varol C, Sagi I: Phagocyte-extracellular matrix crosstalk empowers tumor development and dissemination. FEBS J 2018, 285:734-751

29. Bünemann E, Hoff NP, Buhren BA, Wiesner U, Meller S, Bölke E, Müller-Homey A, Kubitza R, Ruzicka T, Zlotnik A, Homey B: Chemokine ligand-receptor interactions critically regulate cutaneous wound healing. Eur J Med Res 2018, 23:e4

30. Yoshie O, Matsushima K: CCR4 and its ligands: from bench to bedside. Int Immunol 2014, 27:11-20

31. Novak ML, Koh TJ: Phenotypic transitions of macrophages orchestrate tissue repair. Am J Pathol 2013, 183:1352-1363

32. Krzyszczyk P, Schloss R, Palmer A, Berthiaume F: The role of macrophages in acute and chronic wound healing and interventions to promote pro-wound healing phenotypes. Front Physiol 2018, 9:e419

33. Bannon P, Wood S, Restivo T, Campbell L, Hardman MJ, Mace KA: Diabetes induces stable intrinsic changes to myeloid cells that contribute to chronic inflammation during wound healing in mice. Dis Model Mech 2013, 6:1434-1447

34. Kramer JL, Baltathakis I, Alcantara OS, Boldt DH: Differentiation of functional dendritic cells and macrophages from human peripheral blood monocyte precursors is dependent on expression of P21 (WAF1/CIP1) and requires iron. Br J Haematol 2002, 117: 727-734

35. Mirza R, DiPietro LA, Koh TJ: Selective and specific macrophage ablation is detrimental to wound healing in mice. Am J Pathol 2009, $175: 2454-2462$

36. Lin YW, Lee B, Liu PS, Wei LN: Receptor-interacting protein 140 orchestrates the dynamics of macrophage M1/M2 polarization. J Innate Immun 2016, 8:97-107

37. Nelson SM, Lei X, Prabhu KS: Selenium levels affect the IL4-induced expression of alternative activation markers in murine macrophages. J Nutr 2011, 141:1754-1761

38. Kroner A, Greenhalgh AD, Zarruk JG, dos Santos RP, Gaestel M, David S: TNF and increased intracellular iron alter macrophage polarization to a detrimental M1 phenotype in the injured spinal cord. Neuron 2014, 83:1098-1116

39. Zhou Y, Que KT, Zhang Z, Yi ZJ, Zhao PX, You Y, Gong JP, Liu ZJ: Iron overloaded polarizes macrophage to proinflammation 
phenotype through ROS/acetyl-P53 pathway. Cancer Med 2018, 7 : 4012-4022

40. Sindrilaru A, Scharffetter-Kochanek K: Disclosure of the culprits: macrophages-versatile regulators of wound healing. Adv Wound Care 2013, 2:357-368

41. Wlaschek M, Singh K, Sindrilaru A, Crisan D, ScharffetterKochanek K: Iron and iron-dependent reactive oxygen species in the regulation of macrophages and fibroblasts in non-healing chronic wounds. Free Radic Biol Med 2019, 133:262-275

42. Finn AV, Nakano M, Polavarapu R, Karmali V, Saeed O, Zhao X, Yazdani S, Otsuka F, Davis T, Habib A, Narula J, Kolodgie FD, Virmani R: Hemoglobin directs macrophage differentiation and prevents foam cell formation in human atherosclerotic plaques. J Am Coll Cardiol 2012, 59:166-177

43. Agoro R, Taleb M, Quesniaux VF, Mura C: Cell iron status influences macrophage polarization. PLoS One 2018, 13:e0196921

44. Ploeger DT, Hosper NA, Schipper M, Koerts JA, de Rond S, Bank RA: Cell plasticity in wound healing: paracrine factors of M1/M2 polarized macrophages influence the phenotypical state of dermal fibroblasts. Cell Commun Signal 2013, 11:e29

45. Jetten N, Roumans N, Gijbels MJ, Romano A, Post MJ, de Winther MP, van der Hulst RR, Xanthoulea S: Wound administration of M2-polarized macrophages does not improve murine cutaneous healing responses. PLoS One 2014, 9:e102994

46. Nakahigashi K, Kabashima K, Ikoma A, Verkman AS, Miyachi Y, Hara-Chikuma M: Upregulation of aquaporin-3 is involved in keratinocyte proliferation and epidermal hyperplasia. J Invest Dermatol 2011, 131:865-873

47. Buskermolen JK, Roffel S, Gibbs S: Stimulation of oral fibroblast chemokine receptors identifies CCR3 and CCR4 as potential wound healing targets. J Cell Physiol 2017, 232:2996-3005

48. Belperio JA, Dy M, Murray L, Burdick MD, Xue YY, Strieter RM, Keane MP: The role of the Th2 CC chemokine ligand CCL17 in pulmonary fibrosis. J Immunol 2004, 173: 4692-4698

49. Kato T, Saeki H, Tsunemi Y, Shibata S, Tamaki K, Sato S: Thymus and activation-regulated chemokine (TARC)/CC chemokine ligand (CCL) 17 accelerates wound healing by enhancing fibroblast migration. Exp Dermatol 2011, 20:669-674

50. Barros JF, Waclawiak I, Pecli C, Borges PA, Georgii JL, RamosJunior ES, Canetti C, Courau T, Klatzmann D, Kunkel SL, Penido C: Role of chemokine receptor CCR4 and regulatory T cells in wound healing of diabetic mice. J Invest Dermatol 2019, 139: $1161-1170$ 